\title{
Rhipicephalus turanicus: from low numbers to complete establishment in Cyprus. Its possible role as a bridge-vector
}

\author{
D Chochlakis ${ }^{1 *}$, I loannou², B Papadopoulos ${ }^{3}$, Y Tselentis ${ }^{1,3}$, A Psaroulaki ${ }^{3}$ \\ From The 1st Conference on Neglected Vectors and Vector-Borne Diseases (EurNegVec): with Management \\ Committee and Working Group Meetings of the COST Action TD1303 \\ Cluj-Napoca, Romania. 8-11 April 2014
}

We describe herein the abundance of Rhipicephalus turanicus in Cyprus, and its potential role as bridge vector carrying tick-borne pathogens among various hosts.

Following the first recording (1970-72), at low numbers, of $R$. turanicus in Cyprus, two studies took place (March 1999 - March 2001, January 2004 - December 2006), during which, ticks were collected from ruminants (goats, sheep, bovine), dogs and wild mammals (foxes, wild-rabbits and mouflons) from different sites of the island. All ticks were morphologically identified to the species level, washed in $70 \%$ alcohol, rinsed in sterile water, dried on sterile filter paper and triturated individually. Following DNA extraction [QIAampTissue Kit (QIAGEN, Germany)] all ticks were tested for Rickettsia species and Coxiella burnetii by Real-time PCR targeting the gltA gene and IS1111 insertion sequence, respectively, and for Anaplasma species by PCR targeting the 16s rRNA gene. Samples positive for Rickettsia species were further amplified by PCR targeting the ompA and $о m p B$ genes.

Of the 3950 ticks collected, 805 (20.4\%) were identified as $R$. turanicus; this species was the only one, amongst the rest of the ticks $(R$. bursa, $R$. sanguineus, Hyalomma anatolicum excavatum, $H$. marginatum, $H$. m. rufipes, Ixodes gibbosus, I. ventalloi, Haemaphysalis sulcata, H. punctata), that was collected from every host. Of the ticks tested, 125/805 (IR: 15.5\%) were tested positive for Rickettsia species [R. massilliae (83/125), "Candidatus Rickettsia barbariae" (5/125), "Candidatus Rickettsia barbariae genotype Cretocypriensis" (26/125) and "Candidatus Rickettsia tselentii" (11/125)]. The latter Rickettsia species showed a close relationship with $R$. africae, was described for the first time in Cyprus and was identified in $R$. turanicus only disregarding the animal host. Of the 805 ticks, 107 (IR: $13.3 \%$ ) were tested positive for C. burnetii, and 16 (IR: 2\%) were positive for Anaplasma species. Five ticks harboured both Rickettsia species and C. burnetii.

Rhipicephalus turanicus, largely distributed in the Mediterranean sub-region, Africa and Asia, is flexible and adaptable and can be found in a rich variety of hosts (domestic and wild). It has well adapted and spread over a great variety of animal species in Cyprus during the last 40 years and seems to play an importance role as a bridge-vector of tick-borne pathogens. The changing climate, geographical position of Cyprus (wintering area for migratory birds), make the island an important crossover for possible dispersal of ticks and their tick-borne pathogens from East to the West.

\begin{abstract}
Authors' details
${ }^{1}$ Regional Laboratory of Public Health of Crete, Heraklion, Crete, Greece. ${ }^{2}$ Veterinary Services, Nicosia, Cyprus. ${ }^{3}$ Laboratory of Clinical Bacteriology, Parasitology, Zoonoses and Geographical Medicine, University of Crete, Heraklion, Crete, Greece.
\end{abstract}

Published: 1 April 2014

\section{doi:10.1186/1756-3305-7-S1-P11}

Cite this article as: Chochlakis et al:: Rhipicephalus turanicus: from low numbers to complete establishment in Cyprus. Its possible role as a bridge-vector. Parasites \& Vectors 2014 7(Suppl 1):P11. 